\title{
A Two-Stage Study Identifies Two Novel Polymorphisms in PRKAG2 Affecting Metformin Response in Chinese Type 2 Diabetes Patients
}

\author{
Di Xiao ${ }^{1,2, *}$ \\ Jun-Yan Liu ${ }^{3, *}$ \\ Si-Min Zhang ${ }^{4, *}$ \\ Rang-Ru Liu ${ }^{1,5}$ \\ Ji-Ye Yin (ID) ${ }^{1,6}$ \\ Xue-Yao $\mathrm{Han}^{4}$ \\ $\mathrm{Xi} \mathrm{Li}{ }^{1,6}$ \\ Wei Zhang ${ }^{1,6,7}$ \\ Xiao-Ping Chen ${ }^{1,6}$ \\ Hong-Hao Zhou ${ }^{1,6,7}$ \\ Li-Nong Ji ${ }^{4}$ \\ Zhao-Qian Liu ${ }^{1,6,7}$
}

'Department of Clinical Pharmacology, Xiangya Hospital, Central South University, Changsha, People's Republic of China; ${ }^{2}$ Department of pharmacy, Xiangya Hospital, Central South University, Changsha, People's Republic of China; ${ }^{3}$ Department of orthopaedics, Xiangya Hospital, Central South University, Changsha, People's Republic of China; ${ }^{4}$ Department of Endocrinology and Metabolism, The People's Hospital of Peking University, Beijing, People's Republic of China; ${ }^{5}$ Key Laboratory of Tropical Diseases and Translational Medicine of the Ministry of Education \& Hainan Provincial Key Laboratory of Tropical Medicine, Hainan Medical College, Haikou, People's Republic of China; 'Institute of Clinical Pharmacology, Hunan Key Laboratory of Pharmacogenetics, Central South University, Changsha, People's Republic of China; ${ }^{7}$ National Clinical Research Center for Geriatric Disorders, Xiangya Hospital, Central South University, Changsha, People's Republic of China

*These authors contributed equally to this work

Correspondence: Zhao-Qian Liu; Li-NongJi Email liuzhaoqian63@I26.com;

jiln@gmail.com
Objective: Individual differences in glycemic response to metformin in antidiabetic treatment exist widely. Although some associated genetic variations have been discovered, they still cannot accurately predict metformin response. In the current study, we set out to investigate novel genetic variants affecting metformin response in Chinese type 2 diabetes (T2D) patients.

Methods: A two-stage study enrolled 500 T2D patients who received metformin, glibenclamide or a combination of both were recruited from 2009 to 2012 in China. Change of $\mathrm{HbA}_{1 \mathrm{c}}$, adjusted by clinical covariates, was used to evaluate glycemic response to metformin. Selected single nucleotide polymorphisms (SNPs) were genotyped using the Infinium iSelect and/or Illumina GoldenGate genotyping platform. A linear regression model was used to evaluate the association between SNPs and response.

Results: A total of 3739 SNPs were screened in Stage 1, of which 50 were associated with drug response. Except for one genetic variant preferred to affect glibenclamide, the remaining SNPs were subsequently verified in Stage 2, and two SNPs were successfully validated. These were $P R K A G 2$ rs2727528 (discovery group: $\beta=-0.212, P=0.046$; validation group: $\beta=$ -0.269, $P=0.028$ ) and $P R K A G 2$ rs 1105842 (discovery group: $\beta=0.205, P=0.048$; validation group: $\beta=0.273, P=0.025$ ). $C$ allele carriers of rs 2727528 and $C$ allele carriers of rs 1105842 would have a larger difference of $\mathrm{HbA}_{1 \mathrm{c}}$ level when using metformin.

Conclusion: Two variants rs2727528 and rs1105842 in PRKAG2, encoding $\gamma 2$ subunit of AMP-activated protein kinase (AMPK), were found to be associated with metformin response in Chinese T2D patients. These findings may provide some novel information for personalized pharmacotherapy of metformin in China.

Keywords: type 2 diabetes, metformin response, genetic variants, PRKAG2

\section{Introduction}

Type 2 diabetes (T2D) is a common chronic metabolic disease that is harmful to public health. The 2019 International Diabetes Federation (IDF) Diabetes Atlas reported 116.4 million diabetics aged 20 to 79 years in China, making it the country with the highest number of diabetes sufferers in the world. ${ }^{1}$ Among adults in China, the estimated overall prevalence of diabetes is $10.9 \%$, including diagnosed and undiagnosed cases. ${ }^{2}$ Yet, according to the latest epidemiological studies, only $25.8 \%$ of definitely diagnosed patients were receiving antidiabetic therapy, and only about $40 \%$ of patients were under favorable glycemic control. ${ }^{3}$ 
Oral antidiabetic drugs (OADs) can be classified as follows: biguanide (metformin is the only biguanide in general use), second-generation sulfonylureas (SUs), meglitinides, thiazolidinediones (TZDs), $\alpha$-glucosidase inhibitors, dipeptidyl peptidase-4 (DPP-4) inhibitors, and sodium-glucose cotransporter 2 (SGLT2) inhibitors. ${ }^{4}$ Among these, metformin is the most widely used agent owing to its high efficacy, neutral/mild weight loss, low cost, and rare side effect of hypoglycemia. ${ }^{4}$ The American Diabetes Association continued to advise in 2020 that metformin is the preferred initial pharmacologic agent for type 2 diabetes and should be used up to contraindication or intolerance. ${ }^{5}$ Individual differences in glycemic response to metformin in antidiabetic treatment exist widely. Less than half of the T2D patients treated with metformin could reach their $\mathrm{HbA}_{1 \mathrm{c}}$ target $(<7 \%)$ and $30 \%$ experienced an adverse gastrointestinal reaction. ${ }^{6-8}$

Metformin is not metabolized in vivo and is excreted unchanged in urine. Pharmacogenomics of metformin previously focused mainly on genetic variants of its transporters. SNPs within organic cation transporter (OCT) 1-3 (encoded by $S L C 22 A 1, S L C 22 A 2$, SLC22A3, separately $)^{9-12}$ and multidrug and toxin extrusion (MATE) 1/2-k (encoded by SLC47A1/ SLC47A2), ${ }^{13,14}$ plasma monoamine transporter (PMAT; encoded by SLC29A4), ${ }^{15}$ serotonin reuptake transporter (SERT; encoded by $S L C 6 A 4),{ }^{8,16}$ as well as thiamine transporter (THTR-2; encoded by $S L C 19 A 3){ }^{17}$ were reported to take part in the drug disposal process of metformin. Thus, genetic variants of transporters mentioned above probably have an impact on metformin pharmacokinetics, accompanied or not by an influence on pharmacodynamics.

Over the past few decades, about 50 single nucleotide polymorphisms (SNPs) have been found likely to affect its glycemic response, including several genetic variants identified by genome-wide association study (GWAS). These were rs11212617 closed to ATM (a regulator of the target of metformin, AMPK), ${ }^{18}$ rs8192675 in SLC2A2 (the coding gene of a glucose transporter, GLUT2), ${ }^{19}$ rs254271 in PRPF31 (pre-mRNA processing factor 31) and rs2162145 in CPA6 (carboxypeptidase A6). ${ }^{20}$ Taking rs11212617 near $A T M$ as an example, several investigators attempted to conduct replication and meta-analysis of this locus to confirm its influence, but the results were inconsistent. $^{21-23}$ Moreover, these high throughput screening researches were all conducted in a multiethnic population, among which Asians made up a small proportion or were not included.
We used a candidate gene approach, involving thousands of SNPs, to explore the characteristic genetic variants that affect metformin's glycemic response in Chinese T2D patients.

\section{Methods \\ Study Participants}

Data for this study were obtained from two trials. One is the "Glibenclamide" arm of the Xiaoke Pill Trial, described in detail by $\mathrm{Ji}$ et $\mathrm{al}^{24}$. The other is a group of newly diagnosed T2D patients that received metformin monotherapy.

A total of 365 patients were recruited for the "Glibenclamide" arm. Among these, 182 received a combination treatment of metformin plus glibenclamide. We called it the "combination treatment group", or "discovery group". For this group, glycometabolism measurements were assessed at baseline and then every 12 weeks until the trial's termination. Glibenclamide doses were adjusted according to changes of FPG level every four weeks, and metformin doses remained unchanged throughout the trial. Another 183 patients were treatment naïve T2D cases who received glibenclamide monotherapy. We named this as the "glibenclamide monotherapy group", or "exclusion group". Dose adjustment was similar to the above (Trial no. ChiCTR-TRC-08000074).

As for the metformin group, 145 newly diagnosed and drug-naïve T2D patients received metformin monotherapy for 16 weeks. We called it the "metformin monotherapy group" or "validation group". Glycometabolism measurements were evaluated at baseline and at the ending point (Trial no. NCT00778622).

\section{Phenotype Definitions}

Referring to Zhou et $\mathrm{al}^{18}$ we used the change of $\mathrm{HbA}_{1 \mathrm{c}}$ level (on-treatment $\mathrm{HbA}_{1 \mathrm{c}}$ level minus pre-treatment $\mathrm{HbA}_{1 \mathrm{c}}$ level), adjusted by known clinical covariates, as the glycemic response phenotype. On-treatment $\mathrm{HbA}_{1 \mathrm{c}}$ was defined as the minimum recorded $\mathrm{HbA}_{1 \mathrm{c}}$ achieved within 36 weeks after the index date in the "combination treatment group" and "glibenclamide monotherapy group". The covariates included age, sex, weight, serum creatinine (Scr), baseline $\mathrm{HbA}_{1 \mathrm{c}}$ level, and drug doses. If the first four covariates were all available, the creatinine clearance rate (Ccr) would be recommended as a whole instead of being adjusted separately. The Ccr was calculated as the following equation: $(140$-age $) \times$ weight $($ in $\mathrm{kg}) \times(0.85$ if 
female) $/(0.818 *$ Scr (in $\mu \mathrm{mol} / \mathrm{L})$. Drug dose was defined as the average daily dose during the three months prior to the minimum $\mathrm{HbA}_{1 \mathrm{c}}$ being achieved.

\section{Genotyping}

Infinium iSelect HD Custom Genotyping BeadChips and Illumina GoldenGate genotyping platforms were used for patient genotyping. SNPs were primarily selected on the basis of pharmacokinetics and pharmacodynamics, as well as reported disease-related SNPs, such as diabetes, obesity, glucose, and lipid metabolism. The 20 top-ranked GO (Gene Ontology) biological process and KEGG (Kyoto Encyclopedia of Genes and Genomes) Pathway analysis of SNP lists are presented in Figure S1. For iSelect BeadChip, SNP selection was based primarily on the DMET (Drug Metabolizing Enzymes and Transporter; Affymetrix) chip, with some extension. As for GoldenGate BeadChip, SNPs were selected mainly direct to metformin. Genes likely to affect metformin pharmacokinetics and pharmacodynamics, confirmed or speculated, were enrolled. In total, 2986 SNPs were included in the iSelect BeadChip, while 768 SNPs were customed into the GoldenGate BeadChip. For comparability between the two chips, 15 SNPs were customed into both. Because the "combination treatment group" was at the discovery stage, genotyping by both chips was undertaken. Subsequently, the "glibenclamide monotherapy group" used the iSelect chip only because glibenclamide-related genes were involved in this chip, while the "metformin monotherapy group" utilized the GoldenGate Chip for the same reason.

\section{Statistical Analysis}

Before genetic association analysis, SNP quality control (QC) and sample QC were performed in three groups. For each SNP, simultaneously satisfying call rate $\geq 90 \%$ and MAF (minor allele frequency) $\geq 0.05$ and Hardy-Weinberg equilibrium (HWE) test $P$ values $>0.5$ were filtered. For each sample, a genotyping call rate $\geq 90 \%$ was retained for subsequent analyses. Stepwise linear regression was utilized to select clinical covariates of potential effects. Linear regression model was performed to test associations between each SNP and drug efficacy. The Bonferroni correction was used for multiple testing corrections to adjust raw $P$ values. All the above analyses were achieved by using plink 1.07 (http://pngu.mgh.harvard.edu/purcell/ plink/) and SPSS 20.0 (SPSS Inc., Chicago, Illinois, USA).

\section{Results}

\section{Results of SNP Selection and Genotyping} A total of 2986 SNPs and 768 SNPs were included in iSelect BeadChip and GoldenGate BeadChip, separately. The accordance ratio of the 15 reduplicative SNPs was over $98 \%$. In the "combination treatment group", 551 SNPs in iSelect chip and 645 SNPs in GoldenGate Chip passed SNP and sample filtering. In the "glibenclamide monotherapy group", 545 SNPs in iSelect chip passed filtering, while in the "metformin monotherapy group", 644 SNPs in GoldenGate chip passed filtering. The screening process is shown in Table S1.

Table I Demographics of Study Populations

\begin{tabular}{|c|c|c|c|}
\hline Characteristics & $\begin{array}{c}\text { Glibenclamide Monotherapy } \\
\text { Group }\end{array}$ & $\begin{array}{c}\text { Combination Treatment } \\
\text { Group }\end{array}$ & $\begin{array}{c}\text { Metformin Monotherapy } \\
\text { Group }\end{array}$ \\
\hline No.(male/female) & $181(106 / 75)$ & I76(90/86) & I43(84/59) \\
\hline Age(y) & $53.5 \pm 8.5$ & $55.0 \pm 9.4$ & $52.9 \pm 9.9$ \\
\hline Baseline weight(kg) & $67.2 \pm 10.4$ & $67.2 \pm 11.1$ & $73.2 \pm 13.2$ \\
\hline Baseline BMI $\left(\mathrm{kg} / \mathrm{m}^{2}\right)$ & $24.5 \pm 2.5$ & $25.0 \pm 3.1$ & $26.8 \pm 3.4$ \\
\hline Baseline Waist/hip ratio & $0.90 \pm 0.07$ & $0.91 \pm 0.07$ & $0.93 \pm 0.08$ \\
\hline Baseline FPG(mmol/L) & $9.02 \pm 1.57$ & $9.36 \pm 1.71$ & $8.50 \pm 1.80$ \\
\hline Baseline $\mathrm{HbA}_{\mathrm{Ic}}(\%)$ & $8.34 \pm 1.22$ & $8.47 \pm 1.26$ & $8.32 \pm 0.82$ \\
\hline On-treatment $\mathrm{HbA}_{\mathrm{Ic}}(\%)$ & $6.56 \pm 0.90$ & $6.78 \pm 0.99$ & $6.53 \pm 0.54$ \\
\hline Baseline Creatinine $(\mu \mathrm{mol} / \mathrm{L})$ & $74.14 \pm 20.09$ & $70.23 \pm 18.15$ & NA \\
\hline $\begin{array}{l}\text { Glibenclamide daily dose } \\
\text { (mg) }\end{array}$ & $3.75(2.50-5.00)$ & $2.50(2.08-5.00)$ & I \\
\hline Metformin daily dose (mg) & l & $1000(750-1500)$ & $1500(\mid 500-2000)$ \\
\hline
\end{tabular}

Notes: Data are presented as means \pm SD or Median and interquartile range (IQR, 25th and 75th percentile). "NA" stands for missing data. "l" stands for no data for monotherapy patients. 


\section{Characteristics of Study Populations}

Detailed demographics are shown in Table 1. After strictly excluding patients who did not meet the entry criteria but were recruited, there were 176 patients (90 males and 86 females) in the combination treatment group (discovery group), 181 patients (106 males and 75 females) in the glibenclamide monotherapy group (exclusion group) and 143 patients (84 males and 59 females) in the metformin monotherapy group (validation group). Baseline age, weight, BMI, and waist/hip ratio are listed in Table 1. A relatively higher proportion of overweight and obese individuals were observed in the validation group. The baseline FPG levels were, respectively, $9.36 \pm 1.71,9.02 \pm 1.57$, and $8.50 \pm 1.80 \mathrm{mmol} / \mathrm{L}$ in the discovery, exclusion, and validation groups in sequence. The baseline $\mathrm{HbA}_{1 \mathrm{c}}$ levels in turn were $8.47 \pm 1.26,8.34 \pm 1.22$, and $8.32 \pm 0.82 \%$. The on- treatment $\mathrm{HbA}_{1 \mathrm{c}}$ refers to the minimum $\mathrm{HbA}_{1 \mathrm{c}}$ level during visits, and the level was $6.78 \pm 0.99,6.56 \pm 0.90,6.53 \pm 0.54 \%$ in sequence. Correspondingly, medication daily dose was the average daily dose for three months prior to the minimum $\mathrm{HbA}_{1 \mathrm{c}}$ being achieved. For the discovery group, the glibenclamide daily dose was $2.50 \mathrm{mg}(2.08-5.00 \mathrm{mg})$ (IQR, 25th and 75th percentile, the same as below) and the metformin daily dose was $1000 \mathrm{mg}(750-1500 \mathrm{mg})$. For the exclusion group, the glibenclamide daily dose was $3.75 \mathrm{mg}$ $(2.50-5.00 \mathrm{mg})$. For the validation group, the metformin daily dose was $1500 \mathrm{mg}(1500-2000 \mathrm{mg})$.

\section{Results of Genetic Association Analysis}

The integrated workflow is shown in Figure 1.

First, we established the association between genotypes and drug response in the discovery group. We merged

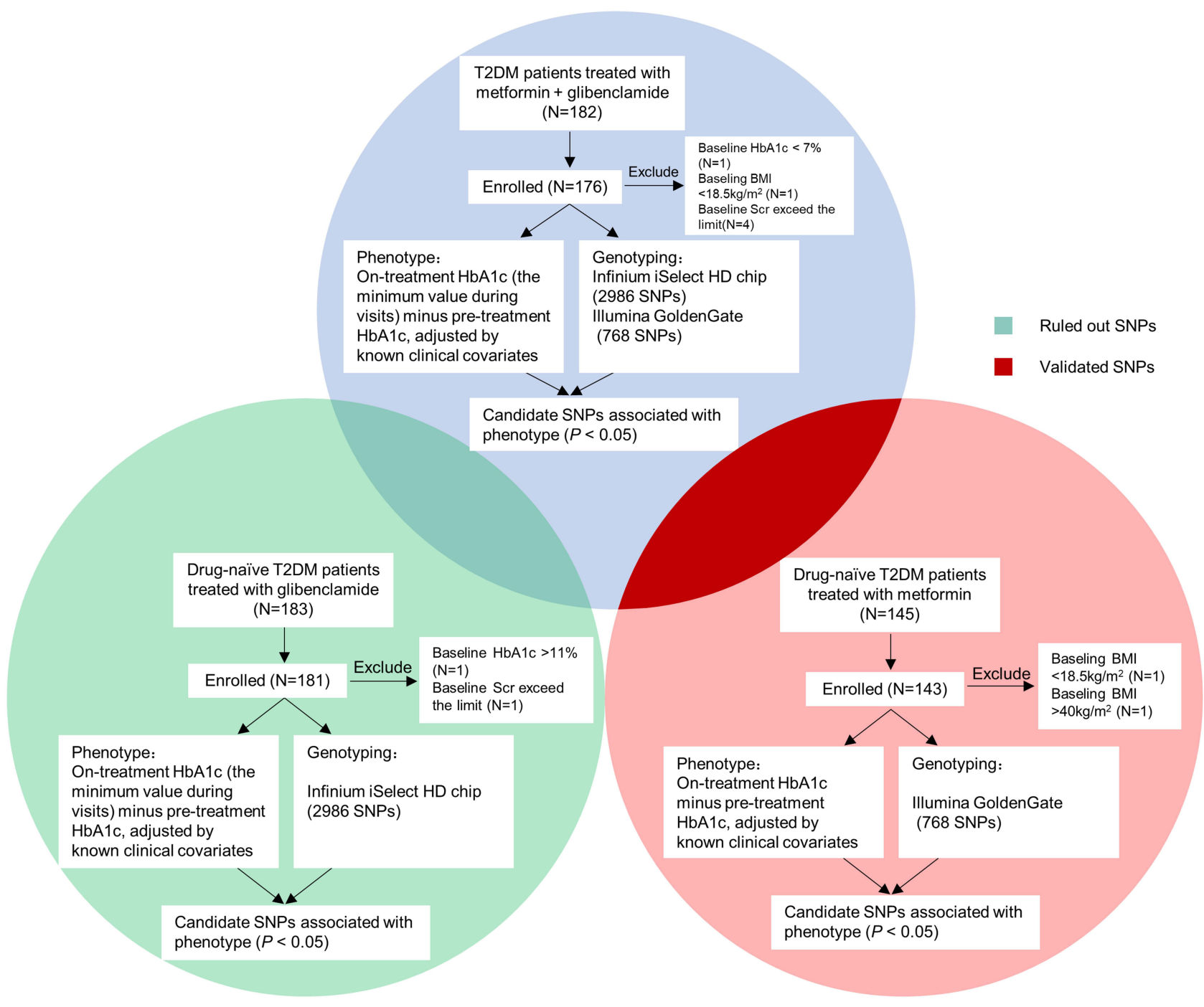

Figure I The design workflow of this clinical study. 
Table 2 SNPs Associated with Phenotype in Discovery Group (Stage I)

\begin{tabular}{|c|c|c|c|c|c|c|c|}
\hline Rs & Chromosome & Position & Nearby Gene & Allele & MAF* & $\boldsymbol{\beta}$ & $P$-value \\
\hline rs34274 & 12 & 109164055 & $\mathrm{ACACB}$ & $\mathrm{A} / \mathrm{G}$ & 0.149 & 0.469 & 0.0017 \\
\hline rs4929949 & 11 & 8583046 & STK33 & $\mathrm{G} / \mathrm{A}$ & 0.422 & 0.300 & 0.0026 \\
\hline rs7438284 & 4 & 69098619 & UGT2B7 & T/A & 0.328 & -0.345 & 0.0034 \\
\hline rs6664 & 3 & $|43| 22898$ & $\mathrm{CHST} 2$ & $\mathrm{~A} / \mathrm{G}$ & 0.386 & -0.333 & 0.0045 \\
\hline rs4l48095 & 21 & 42215846 & $A B C G I$ & $\mathrm{G} / \mathrm{A}$ & 0.155 & 0.413 & 0.0047 \\
\hline rs4726084 & 7 & 151687417 & PRKAG2 & $\mathrm{A} / \mathrm{G}$ & 0.239 & 0.336 & 0.0056 \\
\hline rs972283 & 7 & 130782095 & LOCI05375508 & $\mathrm{A} / \mathrm{G}$ & 0.290 & -0.303 & 0.0076 \\
\hline rs4646440 & 7 & 99763247 & CYP3A4 & $\mathrm{A} / \mathrm{G}$ & 0.251 & 0.321 & 0.0093 \\
\hline rs 1423096 & 19 & 7674291 & RETN & $\mathrm{A} / \mathrm{G}$ & 0.172 & 0.346 & 0.0101 \\
\hline rs61II5830 & 20 & 396582 & TRIB3 & $\mathrm{A} / \mathrm{G}$ & 0.283 & -0.298 & 0.0105 \\
\hline rs2237988 & 11 & 17422587 & $\mathrm{ABCC} 8$ & $\mathrm{~A} / \mathrm{G}$ & 0.230 & -0.314 & 0.0111 \\
\hline rs229964I & 11 & $174 \mid 9443$ & $\mathrm{ABCC} 8$ & $\mathrm{C} / \mathrm{G}$ & 0.171 & 0.366 & 0.0125 \\
\hline rs7483 & 1 & 109737079 & GSTM3 & $\mathrm{G} / \mathrm{A}$ & 0.261 & 0.321 & 0.0133 \\
\hline rs909530 & I & $17|1| 4034$ & $\mathrm{FMO3}$ & $\mathrm{A} / \mathrm{G}$ & 0.332 & 0.273 & 0.0133 \\
\hline rs4402960 & 3 & 185793899 & IGF2BP2 & $\mathrm{A} / \mathrm{C}$ & 0.279 & -0.291 & 0.0170 \\
\hline rs12233719 & 4 & 69096731 & UGT2B7 & $\mathrm{A} / \mathrm{C}$ & 0.149 & 0.317 & 0.0170 \\
\hline rs730570 & 14 & 100676553 & LOCI05370668 & $\mathrm{A} / \mathrm{G}$ & 0.171 & 0.330 & 0.0171 \\
\hline rs4755228 & 11 & 44107740 & EXT2 & $\mathrm{A} / \mathrm{C}$ & 0.320 & -0.275 & 0.0179 \\
\hline rs I 2924026 & 16 & 15991796 & $\mathrm{ABCCl}$ & $\mathrm{G} / \mathrm{A}$ & 0.055 & -0.559 & 0.0186 \\
\hline rs4l48330 & 16 & |59479|| & $\mathrm{ABCCl}$ & $\mathrm{G} / \mathrm{A}$ & 0.437 & 0.236 & 0.0208 \\
\hline rs10906II5 & 10 & 12272998 & $\mathrm{CDCl} 23$ & $\mathrm{G} / \mathrm{A}$ & 0.372 & -0.258 & 0.0214 \\
\hline rs4I484I6 & 17 & 50676062 & $\mathrm{ABCC} 3$ & $\mathrm{~A} / \mathrm{G}$ & 0.140 & 0.353 & 0.0214 \\
\hline rs2236135 & 14 & 23126512 & SLC7A8 & $\mathrm{G} / \mathrm{A}$ & 0.455 & 0.240 & 0.0225 \\
\hline rsl7|4987 & 17 & 37386072 & CI7orf78 & $\mathrm{C} / \mathrm{G}$ & 0.426 & 0.243 & 0.0231 \\
\hline rs6975294 & 7 & 151641118 & PRKAG2 & $\mathrm{A} / \mathrm{T}$ & 0.216 & 0.287 & 0.0233 \\
\hline rs7|36445 & 12 & 21171814 & SLCOIBI & $\mathrm{G} / \mathrm{A}$ & 0.477 & -0.258 & 0.0258 \\
\hline rs10916824 & 1 & 20592419 & CDA & $G / A$ & 0.097 & -0.407 & 0.0259 \\
\hline rs5050 & 1 & $2307|4| 40$ & AGT & $\mathrm{C} / \mathrm{A}$ & 0.171 & -0.298 & 0.0301 \\
\hline rs2297322 & 13 & 98723927 & SLCI5AI & $\mathrm{A} / \mathrm{G}$ & 0.412 & -0.224 & 0.0306 \\
\hline rs2453594 & 17 & 19581638 & SLC47AI & $G / A$ & 0.244 & 0.276 & 0.0308 \\
\hline rs4952986 & 2 & 43347159 & THADA & $\mathrm{A} / \mathrm{G}$ & 0.344 & -0.232 & 0.0308 \\
\hline rs864745 & 7 & $28 \mid 40937$ & JAZFI & $\mathrm{G} / \mathrm{A}$ & 0.233 & -0.256 & 0.0331 \\
\hline rs|3233587 & 7 & 151832150 & PRKAG2 & $\mathrm{A} / \mathrm{G}$ & 0.376 & -0.240 & 0.0331 \\
\hline rs3782905 & 12 & 47872384 & VDR & $\mathrm{C} / \mathrm{G}$ & 0.181 & 0.285 & 0.0334 \\
\hline rs21209| & 16 & 16142793 & $\mathrm{ABCCl}$ & $\mathrm{G} / \mathrm{A}$ & 0.219 & 0.275 & 0.0349 \\
\hline rs I I 28977 & 1 & 165419892 & $R \times R G$ & $\mathrm{~A} / \mathrm{G}$ & 0.159 & -0.306 & 0.0353 \\
\hline rs13959 & 9 & 72930966 & ALDHIAI & $\mathrm{A} / \mathrm{G}$ & 0.440 & -0.218 & 0.0357 \\
\hline rs4726070 & 7 & 151631132 & PRKAG2 & $\mathrm{A} / \mathrm{G}$ & 0.299 & 0.234 & 0.0358 \\
\hline rs375I889 & 16 & 1220055 & CACNAIH & $\mathrm{G} / \mathrm{A}$ & 0.085 & 0.391 & 0.0365 \\
\hline rs3755740 & 3 & 143118124 & CHST2 & $\mathrm{A} / \mathrm{G}$ & 0.409 & -0.225 & 0.0385 \\
\hline rs 1800545 & 10 & III077780 & ADRA2A & $\mathrm{A} / \mathrm{G}$ & 0.179 & 0.294 & 0.0386 \\
\hline rs 1531343 & 12 & 65781114 & RPSAP52 & $C / G$ & 0.106 & 0.354 & 0.0396 \\
\hline rs3814573 & 10 & 113138334 & TCF7L2 & $\mathrm{G} / \mathrm{A}$ & 0.332 & -0.240 & 0.0401 \\
\hline rsII32054 & 19 & 48599142 & SULT2BI & $\mathrm{A} / \mathrm{G}$ & 0.347 & -0.226 & 0.0440 \\
\hline rs 12518099 & 5 & 90250292 & CETN3 & $\mathrm{G} / \mathrm{A}$ & 0.425 & 0.218 & 0.0446 \\
\hline rs2727528 & 7 & 151653366 & PRKAG2 & C/A & 0.379 & -0.212 & $0.046 I$ \\
\hline rsl645694 & 19 & 41094903 & CYP2AI3 & $\mathrm{A} / \mathrm{G}$ & 0.080 & 0.371 & 0.0470 \\
\hline rsII 05842 & 7 & 151667178 & PRKAG2 & $A / C$ & 0.399 & 0.205 & 0.0476 \\
\hline rs6952398 & 7 & 151699167 & PRKAG2 & $\mathrm{G} / \mathrm{A}$ & 0.110 & 0.334 & 0.0492 \\
\hline rs730947 & 2 & 218838575 & PRKAG3 & $\mathrm{C} / \mathrm{A}$ & 0.239 & -0.247 & 0.0495 \\
\hline
\end{tabular}

Notes: *Minor allele frequency is calculated from the subjects; Position is based on GRCh38. pl2; Genetic variants with $P$ value less than 0.05 in both two stages are presented in bold.

Abbreviations: MAF, minor allele frequency; $\beta$, beta coefficient. 
genotyping data of two platforms and redid SNP and sample QC. 1245 SNPs passed filter, including 14 reduplicative loci between the two platforms, so that the number of enrolled SNPs was 1231. After adjustment for baseline $\mathrm{HbA}_{1 \mathrm{c}}$ level, Ccr, and medication daily dose, 50 SNPs were found to be associated with the change of $\mathrm{HbA}_{1 \mathrm{c}}$ value $(P<0.05$, shown in Table 2). Among these probably positive loci, $60 \%$ were from the GoldenGate chip.

Next, associations between genotype and phenotype in the exclusion group were analyzed. 19 of 545 SNPs were found related to glibenclamide response $(P<0.05$, shown in Table 3), among which was rs1800545 in $A D R A 2 A$ (adrenoceptor alpha $2 \mathrm{~A}$ ) with $P$ value less than 0.05 in both groups above. Our preference is that this variant is the most likely to affect glibenclamide response.

Because most SNPs found in the discovery group were derived from the GoldenGate chip, only GoldenGate genotyping was performed on validation group patients using metformin monotherapy. In this group, 27 of 644 SNPs were found to be correlated with metformin glucoselowering efficacy $(P<0.05$, shown in Table 4$)$. Compared with SNPs identified in the discovery group, two variants of the PRKAG2 (protein kinase AMP-activated noncatalytic subunit gamma 2) gene were validated (bold in Tables 2 and 4). One was PRKAG2 rs2727528 (discovery group: $\beta=-0.212, P=0.046$; validation group: $\beta=-0.269$,
$P=0.028$ ). The other was $P R K A G 2$ rs 1105842 (discovery group: $\beta=0.205, P=0.048$; validation group: $\beta=0.273$, $P=0.025)$. C allele carriers $(\mathrm{W} / \mathrm{M}+\mathrm{M} / \mathrm{M}, \mathrm{W}=$ wild type; $\mathrm{M}=$ mutation type) of rs2727528 and $\mathrm{C}$ allele carriers (W/ $\mathrm{W}+\mathrm{W} / \mathrm{M})$ of rs1105842 would have a larger difference of $\mathrm{HbA}_{1 \mathrm{c}}$ level when using metformin (shown in Figure 2). Meanwhile, we were concerned that in the metformin monotherapy group, there were 5 SNPs located in the PRKAG2 gene found to be associated with metformin response. Except for the two SNPs mentioned above, the other three were rs1029946 $(\beta=0.306, \quad P=0.001)$, rs6964824 $(\beta=-0.347, \quad P=0.013), \quad$ and $\quad$ rs2727551 $(\beta=0.296, \quad P=0.042)$. Linkage disequilibrium analysis showed that the linkage among the five SNPs was relatively low (shown in Figure 3). In addition, rs11212617 near C11orf65 or ATM, identified by the first metformin GWAS, was repeated in the metformin monotherapy group $(\beta=-0.255, P=0.035)$, while $\mathrm{C}$ allele carriers benefited more in our research.

\section{Discussion}

To our knowledge, the current study is the first to use highthroughput genotyping chips to identify candidate SNPs, which may affect metformin response in Chinese T2D patients through a two-stage study. Three groups totaling 500 patients met the final selection criteria and were

Table 3 SNPs Associated with Phenotype in Exclusion Group (Stage I)

\begin{tabular}{|c|c|c|c|c|c|c|c|}
\hline Rs & Chromosome & Position & Nearby Gene & Allele & MAF* & $\beta$ & $P$-value \\
\hline rs953062 & 6 & 46658616 & SLC25A27 & $\mathrm{G} / \mathrm{A}$ & 0.282 & -0.339 & 0.0051 \\
\hline rs2156609 & 18 & 45667036 & $\mathrm{SLCl} 14 \mathrm{~A} 2$ & $\mathrm{C} / \mathrm{G}$ & 0.376 & 0.295 & 0.0059 \\
\hline rs2229523 & 6 & 85489515 & NT5E & $A / G$ & 0.403 & 0.314 & 0.0060 \\
\hline rs7797834 & 7 & 92113836 & CYP5IAI & $\mathrm{G} / \mathrm{A}$ & 0.193 & 0.339 & 0.0127 \\
\hline rsI05089I & 2 & $1380|4| 90$ & HNMT & G/A & 0.287 & -0.266 & 0.0163 \\
\hline rs721950 & 8 & 20181826 & SLCI8AI & $\mathrm{A} / \mathrm{C}$ & 0.180 & -0.310 & 0.0171 \\
\hline rs938|468 & 6 & 46657537 & SLC25A27 & $A / G$ & 0.425 & -0.233 & 0.0285 \\
\hline rs 1800545 & 10 & III 077780 & ADRA2A & $A / G$ & 0.160 & 0.300 & 0.0297 \\
\hline rs3743369 & 15 & 92164339 & SLCO3AI & $A / G$ & 0.224 & 0.255 & 0.0308 \\
\hline rs2295490 & 20 & 388261 & TRIB3 & $\mathrm{G} / \mathrm{A}$ & 0.233 & 0.263 & 0.0335 \\
\hline rs47I5333 & 6 & $5280445 \mathrm{I}$ & GSTAI & $\mathrm{A} / \mathrm{C}$ & 0.467 & 0.213 & 0.0340 \\
\hline rs324420 & 1 & 46405089 & FAAH & $\mathrm{A} / \mathrm{C}$ & 0.130 & -0.336 & 0.0365 \\
\hline rs I7707947 & 5 & 16877635 & MYOIO & $A / G$ & 0.113 & 0.343 & 0.0379 \\
\hline rs2952I5I & 17 & 39672243 & PGAP3 & G/A & 0.459 & -0.219 & 0.0398 \\
\hline rs2072330 & 17 & $1974 \mid 159$ & ALDH3AI & $\mathrm{T} / \mathrm{A}$ & 0.243 & 0.249 & 0.0399 \\
\hline rs373I596 & 2 & 226797473 & IRSI & $\mathrm{G} / \mathrm{A}$ & 0.052 & 0.486 & 0.0416 \\
\hline rs4646227 & 13 & $98706 \mid 47$ & SLCI5AI & $C / G$ & 0.072 & 0.424 & 0.0454 \\
\hline rsII I70903 & 7 & 95397015 & PON3 & $\mathrm{G} / \mathrm{A}$ & 0.204 & 0.259 & $0.046 I$ \\
\hline rs2049900 & 7 & 92109474 & AKAP9 & $\mathrm{G} / \mathrm{C}$ & 0.343 & -0.214 & 0.0475 \\
\hline
\end{tabular}

Notes: *Minor allele frequency is calculated from the subjects; Position is based on GRCh38. pI2.

Abbreviations: MAF, minor allele frequency; $\beta$, beta coefficient. 
Table 4 SNPs Associated with Phenotype in Validation Group (Stage 2)

\begin{tabular}{|c|c|c|c|c|c|c|c|}
\hline Rs & Chromosome & Position & Nearby Gene & Allele & MAF* & $\beta$ & $P$-value \\
\hline rs215096 & 16 & 15961589 & $\mathrm{ABCCl}$ & $\mathrm{G} / \mathrm{A}$ & 0.147 & 0.465 & 0.0064 \\
\hline rs 1029946 & 7 & 151578720 & PRKAG2 & $\mathrm{G} / \mathrm{A}$ & 0.462 & 0.306 & 0.0096 \\
\hline rs46075I7 & 7 & 44196069 & GCK & $\mathrm{A} / \mathrm{G}$ & 0.175 & 0.399 & 0.0103 \\
\hline rs6964824 & 7 & $151654 \mid 46$ & PRKAG2 & $\mathrm{G} / \mathrm{A}$ & 0.206 & -0.347 & 0.0127 \\
\hline rs4l48622 & 11 & 17427455 & $\mathrm{ABCC} 8$ & $\mathrm{~A} / \mathrm{G}$ & 0.133 & 0.404 & 0.0146 \\
\hline rs 10423928 & 19 & 45679046 & GIPR & $\mathrm{T} / \mathrm{A}$ & 0.220 & 0.336 & 0.0151 \\
\hline rs2292772 & 12 & 21892837 & ABCC9 & $\mathrm{G} / \mathrm{A}$ & 0.210 & -0.331 & 0.0196 \\
\hline rs3746103 & 19 & 1233682 & CBARP & $\mathrm{A} / \mathrm{G}$ & 0.115 & -0.403 & 0.0231 \\
\hline rs7615776 & 3 & 126341774 & KLFI5 & $\mathrm{A} / \mathrm{G}$ & 0.325 & -0.281 & 0.0232 \\
\hline rs10498769 & 6 & 46649581 & CYP39AI & $\mathrm{C} / \mathrm{G}$ & 0.126 & -0.417 & 0.0238 \\
\hline rs915654 & 6 & 31570720 & LTA & $\mathrm{T} / \mathrm{A}$ & 0.479 & 0.268 & 0.0242 \\
\hline rs7301876 & 12 & 21881686 & $A B C C 9$ & $\mathrm{~A} / \mathrm{G}$ & 0.231 & -0.306 & 0.0243 \\
\hline rsII 05842 & 7 & 151667178 & PRKAG2 & $A / C$ & 0.423 & 0.273 & 0.0250 \\
\hline rsI5I4I75 & 1 & 74525960 & TNNI3K & $\mathrm{G} / \mathrm{A}$ & 0.248 & -0.315 & 0.0251 \\
\hline rs3856806 & 3 & 12434058 & PPARG & $\mathrm{A} / \mathrm{G}$ & 0.245 & 0.296 & 0.0263 \\
\hline rs2727528 & 7 & 151653366 & PRKAG2 & C/A & $0.38 I$ & -0.269 & $0.028 I$ \\
\hline rs340874 & I & 213985913 & PROXI & $\mathrm{G} / \mathrm{A}$ & 0.402 & 0.275 & 0.0313 \\
\hline rs2299869 & 6 & 35415655 & PPARD & $\mathrm{A} / \mathrm{G}$ & 0.157 & $-0.34 \mathrm{I}$ & 0.0325 \\
\hline rsI552224 & 11 & 72722053 & ARAPI & $\mathrm{C} / \mathrm{A}$ & 0.077 & 0.463 & 0.0345 \\
\hline rs 1800796 & 7 & 22726627 & IL6 & $C / G$ & 0.308 & -0.271 & 0.0349 \\
\hline rs|875796 & 3 & 12402158 & PPARG & $\mathrm{G} / \mathrm{A}$ & 0.450 & 0.254 & 0.0353 \\
\hline rsII212617 & 11 & $1084 \mid 2434$ & Cllorf65 & $\mathrm{A} / \mathrm{C}$ & 0.385 & -0.255 & 0.0353 \\
\hline rs24I7940 & 12 & 2086494 I & SLCOIB3 & $\mathrm{A} / \mathrm{G}$ & 0.140 & -0.334 & 0.0363 \\
\hline rs6436094 & 2 & 218822874 & PRKAG3 & $G / A$ & 0.465 & -0.239 & 0.0422 \\
\hline rs272755I & 7 & 151694567 & PRKAG2 & $\mathrm{A} / \mathrm{G}$ & 0.203 & 0.296 & 0.0423 \\
\hline rs65II64 & 6 & 160160342 & SLC22AI & $\mathrm{G} / \mathrm{A}$ & 0.413 & -0.221 & 0.0460 \\
\hline rs|0838738 & 11 & $4764 \mid 497$ & $\mathrm{MTCH} 2$ & $\mathrm{G} / \mathrm{A}$ & 0.266 & -0.287 & 0.0467 \\
\hline
\end{tabular}

Notes: *Minor allele frequency is calculated from the subjects; Position is based on GRCh38. pl2; Genetic variants with $P$ value less than 0.05 in both two stages are presented in bold.

Abbreviations: MAF, minor allele frequency; $\beta$, beta coefficient.

analyzed. Previous studies on metformin pharmacogenomics were mostly carried out in patients receiving combination therapy, with at least one more antidiabetic drug being added to metformin. Even if subjects were metformin monotherapy patients, or considering monotherapy patients as a subgroup, the sample size was usually relatively small. This was understandable for at least two reasons. First, many T2D patients have progressed to such a degree that a single drug could not well control at the time of diagnosis. That is why we emphasize screening for diabetes. Second, as described in the introduction, a proportion of patients do not respond well to metformin or cannot tolerate its side effects. To cripple interference from the combined drugs, we individually recruited commensurate patients for treatment with the specified antidiabetic drug. We validated our results at the discovery stage in metformin monotherapy patients. All the above was to strengthen the credibility of verified SNPs in affecting metformin response in Chinese T2D patients.

In the discovery group, we screened out 50 SNPs nominally associated with the change of $\mathrm{HbA}_{1 \mathrm{c}}$ value. Although the one with the lowest $P$ value $\left(10^{-3}\right.$ level $)$ did not pass the Bonferroni test (Bonferroni $P$ value should be less than $4.06 \times 10^{-5}$ ), potential impacts could be masked. Furthermore, due to the combination of metformin and glibenclamide, we did not know the contribution of each drug in glucose lowering. To minimize the influence, 19 SNPs were identified for association with glibenclamide response in the glibenclamide monotherapy group. It was not surprising that some of them were located in or near "known" genes to affect pharmacokinetics or pharmacodynamics of sulfonylureas, such as IRS1 ${ }^{25}{ }^{25}$ P 51A1, ${ }^{26}$ ADRA2A, ${ }^{27}$ and so on. Due to racial differences in allele frequency, some crucial variants of 
A

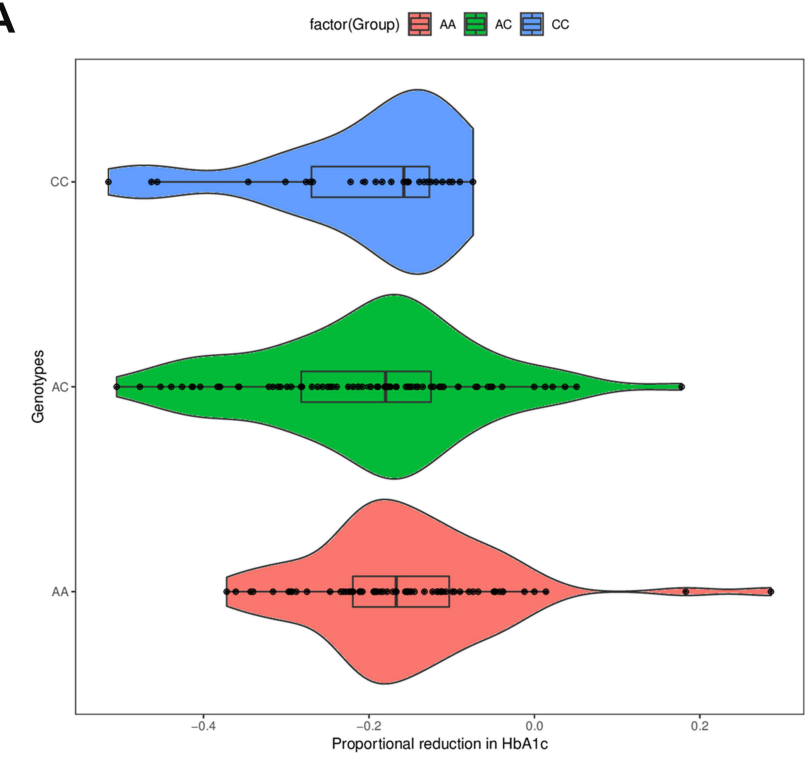

C

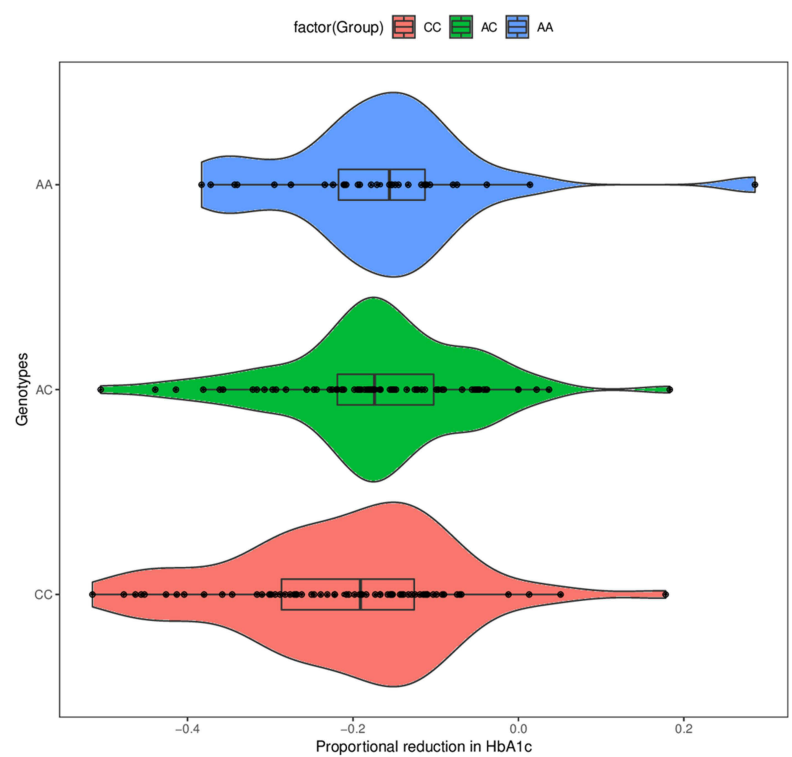

B

factor(Group) 固 ${ }^{A A}$ 目 ${ }^{A C}$ 圄 ${ }^{\mathrm{CC}}$

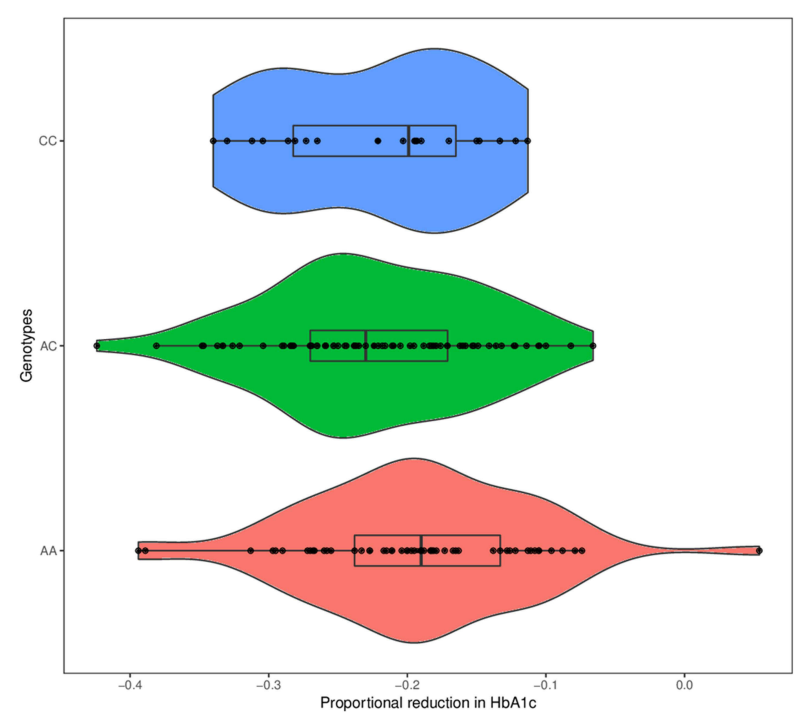

D

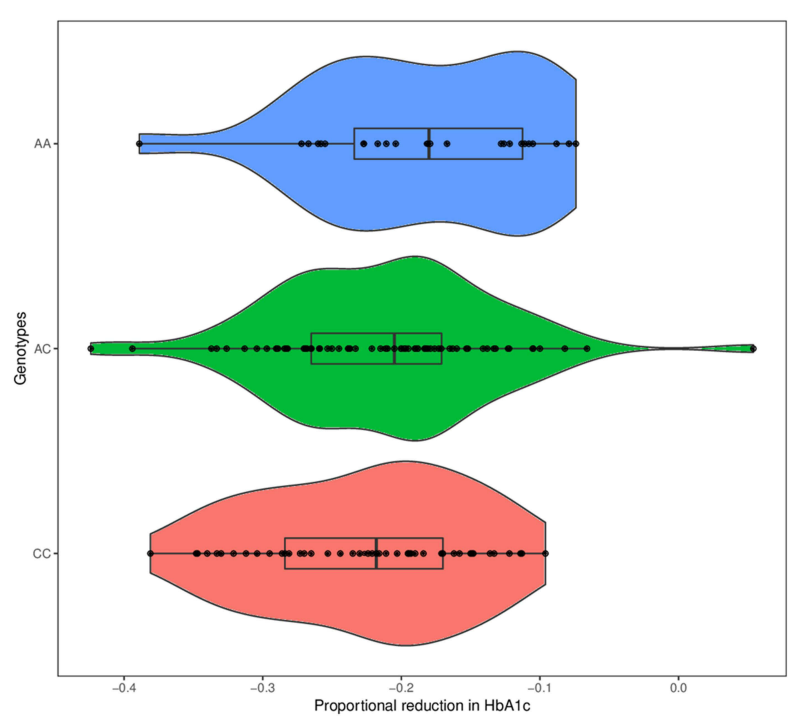

Figure 2 Proportional reduction in $\mathrm{HbA}_{\mathrm{Ic}}$ by PRKAG2 rs2727528 and rs 105842 genotypes as represented by violin plots. Proportional reduction in $\mathrm{Hb} \mathrm{A}_{\mathrm{Ic}}$ was calculated as (on-treatment $\mathrm{HbA}_{\mathrm{Ic}}$ level minus pre-treatment $\mathrm{HbA}_{\mathrm{Ic}}$ level)/pre-treatment $\mathrm{HbA}_{\mathrm{Ic}}$ level. (A) Proportional reduction in $\mathrm{Hb}_{\mathrm{Ic}}$ among PRKAG2 rs2727528 different genotypes in discovery group; (B) Proportional reduction in $\mathrm{HbA}_{\mathrm{lc}}$ among PRKAG2 $\mathrm{rs} 2727528$ different genotypes in validation group; (C) Proportional reduction in HbA $\mathrm{A}_{\mathrm{lc}}$ among PRKAG2 rsI 105842 different genotypes in discovery group; (D) Proportional reduction in $\mathrm{HbA}_{\mathrm{Ic}}$ among PRKAG2 rs I 105842 different genotypes in validation group.

sulfonylureas like *2 variant (Arg144Cys, rs 1799853), *3 variant (Ile359Leu, rs1057910) of $C Y P 2 C 9^{28}$ were rare mutations in Chinese patients, so that they either were not selected in the genotyping chip originally, or did not pass MAF filtering. By comparing the results of the two groups above, one repeated locus was regarded as associated with glibenclamide, but not metformin. Over $60 \%$ of the remaining 49 SNPs came from the GoldenGate chip, which was targeted at metformin's intracorporal process and efficacy. Thus, we decided to verify the remaining
SNPs using only the GoldenGate chip. A total of 27 SNPs with a raw $P$ value less than 0.05 and PRKAG2 rs2727528 and rs1105842 were duplicated in both discovery and validation groups. Further analysis indicated that C allele carriers of rs2727528 and C allele carriers of rs1105842 would have a larger difference of $\mathrm{HbA}_{1 \mathrm{c}}$ level when using metformin. This could mean that patients with prepotent genotype will obtain more benefit from metformin in glucose control. Meanwhile, we were concerned that in the metformin monotherapy group, five SNPs 
A

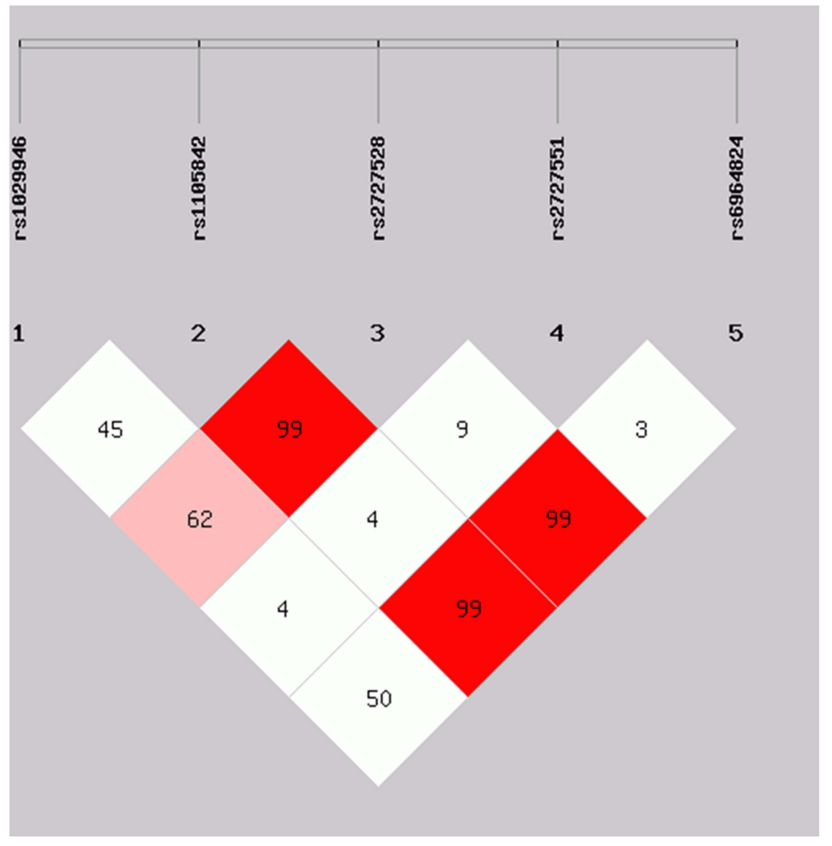

B

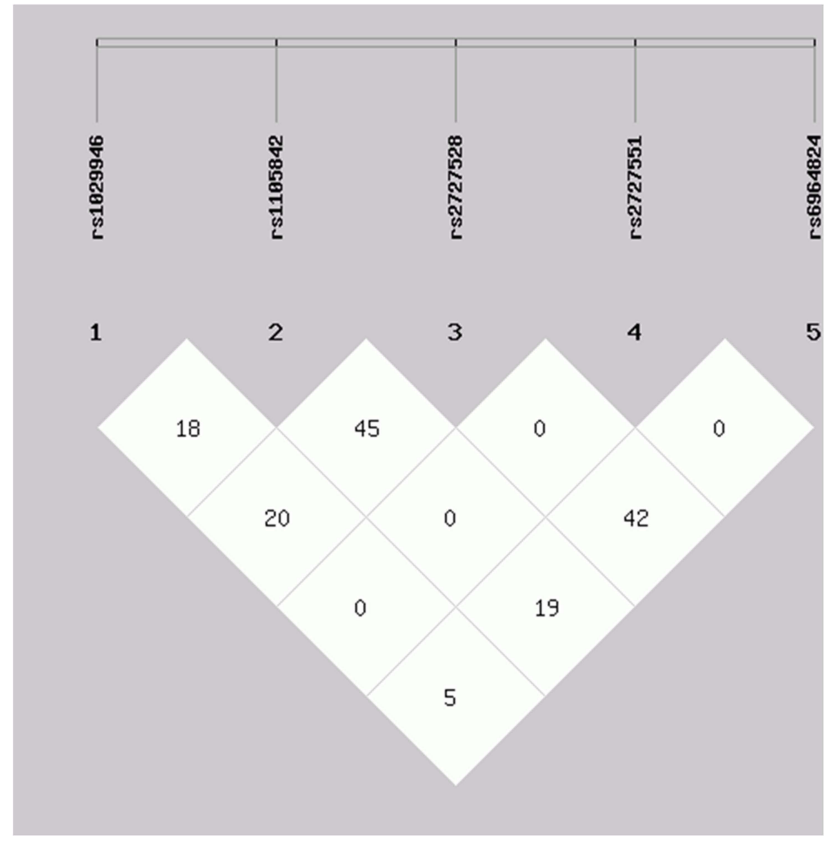

Figure 3 Linkage disequilibrium analysis of 5 SNPs (rs 1029946, rs I 105842, rs2727528, rs272755I, rs6964824) in PRKAG2 in validation group. (A) D' of the 5 SNPs in PRKAG2; (B) $r^{2}$ of the 5 SNPs in PRKAG2.

located in the PRKAG2 gene were nominally associated with metformin response, and were in poor linkage with each other. This suggests that PRKAG2 and its variants may contribute more to metformin efficacy than we recognize.

Metformin has been shown to act via both AMPactivated protein kinase (AMPK)-dependent and AMPKindependent mechanisms. ${ }^{29}$ AMPK is a heterotrimeric complex consisting of a catalytic subunit ( $\alpha$, encoded by PRKAA1 and PRKAA2) and two regulatory subunits ( $\beta$, encoded by PRKAB1 and PRKAB2; $\gamma$, encoded by PRKAG1, PRKAG2, and PRKAG3). ${ }^{30,31}$ The $\gamma$-subunit harbors nucleotide-binding sites and plays an important role in AMPK regulation in response to cellular energy levels. In mammals, there are three isoforms of the $\gamma$ subunit, and these respond differently to regulation by nucleotides. ${ }^{32,33} \mathrm{~A}$ recent study has further reported that humans carrying the R302Q mutation in $\gamma 2$ have increased adiposity and slightly raised fasting glucose levels compared with unaffected individuals, owing to chronic activation of $\gamma 2$ AMPK when mutation exists. ${ }^{34}$ This suggests that mutation could change the state of activation. Genome-wide association studies show that PRKAG2 is significantly associated with diabetes incidence. ${ }^{35}$ In addition, methylation signatures of $\operatorname{cg} 24061580$ (PRKAG2) correlate with insulin resistance. ${ }^{36}$ Polymorphisms in encoding genes of other subunits, PRKAAl (encode $\alpha 1$ ), PRKAA2 (encode $\alpha 2$ ), and PRKAB2 (encode $\beta 2$ ), have been found to affect metformin glucose-lowering effect. ${ }^{37}$ However, PRKAG2 has been extensively studied mainly for its mutations, which could cause human cardiomyopathy characterized by hypertrophy, Wolff-Parkinson -White syndrome, conduction system disease, and glycogen storage in the myocardium. ${ }^{38}$ Recent studies have revealed the molecular pathogenesis of cardiac abnormality owing to PRKAG2 mutation. PRKAG2 mutant patients and model mice displayed anomalous atrioventricular conduction related to cardiac glycogen overload. Most likely, the increased AMPK activity caused by active mutation enhanced glycogen synthesis through robust glucose uptake. ${ }^{39,40}$ That is, glucose-6-phosphate and the abundant substrate functioned as allosteric activators of glycogen synthase, thus promoting the influx of glucose by AMPK activation to synthesize glycogen. However, because of insulin deficiency and glucagon-induced insulin resistance, diabetics cannot store glucose as liver glycogen, either directly (glycogen synthesis from dietary glucose after meals) or indirectly (glycogen synthesis from "de novo" synthesis of glucose).

Our study found that $P R K A G 2$ rs2727528 and rs 1105842 could affect the hypoglycemic effect of metformin in Chinese Han T2D patients. We speculate that the mutation 
in PRKAG2 might change the conformation or activity of $\gamma 2$ AMPK, thus altering the rate of gluconeogenesis, glycogen cycling, and hepatic glucose output. Coincidentally, metformin acts primarily by decreasing hepatic glucose output, largely by inhibiting gluconeogenesis. ${ }^{41}$ The interaction between metformin and PRKAG2 mutation is fascinating. However, our hypothesis needs to be verified by cell and animal experiments.

There were certain limitations to our study. First, superabundant trivial loci were enrolled when designing the genotyping chip, especially those with very low allele frequency in Chinese people. Second, due to differences in visit times, only 16-week glycometabolism and lipometabolism measures were collected in the metformin monotherapy group. A different course of treatment compared with the discovery group may mask the effects of some meaningful gene variants.

\section{Conclusion}

Nevertheless, this is progressive research with a more rigorous grouping and a larger population to screen genetic variants that could affect metformin response in Chinese T2D patients. By correlating the change of $\mathrm{HbA}_{1 \mathrm{c}}$ levels with thousands of related SNPs, we found that PRKAG2 rs2727528 and rs1105842 polymorphisms may affect metformin response in Chinese T2D patients. The mechanisms of their influence need further research.

\section{Data Sharing Statement}

The data used to support the findings of this study are available from the corresponding authors on reasonable request (Professor Zhao-Qian Liu, E-mail: liuzhaoqian63@126.com and Professor Li-Nong Ji, Email: jiln@gmail.com).

\section{Ethics Approval and Informed Consent}

The study was approved by the Ethics Committee of Xiangya School of Medicine, Central South University (Changsha, Hunan, China) (CTXY-110002-5), and was performed in accordance with the Helsinki Declaration. Written informed consent was provided by all subjects.

\section{Acknowledgments}

We thank all the participants in this clinical study. This work was supported by the National High-tech R\&D Program of China (863 Program) (2009AA022704,
2012AA02A517), National Natural Science Foundation of China (81703620, 81874327), Hunan Provincial Natural Science Foundation of China Grant (2018JJ3845), and Hainan Province Key Research and Development Project (ZDYF2016128).

\section{Disclosure}

The authors declared no competing interests for this work.

\section{References}

1. Saeedi P, Petersohn I, Salpea P, et al. Global and regional diabetes prevalence estimates for 2019 and projections for 2030 and 2045: results from the International Diabetes Federation Diabetes Atlas, 9 (th) edition. Diabetes Res Clin Pract. 2019;157:107843. doi:10.1016/ j.diabres.2019.107843

2. Wang L, Gao P, Zhang M, et al. Prevalence and ethnic pattern of diabetes and prediabetes in China in 2013. JAMA. 2017;317:2515-2523. doi:10.1001/jama.2017.7596

3. Xu Y. Prevalence and control of diabetes in Chinese adults. JAMA. 2013;310:948-959. doi:10.1001/jama.2013.168118

4. Upadhyay J. Pharmacotherapy of type 2 diabetes: an update. Metabolism. 2018;78:13-42.

5. American Diabetes Association.9. Pharmacologic approaches to glycemic treatment: standards of medical care in diabetes-2020. Diabetes Care. 2020;43:S98-S110. doi:10.2337/dc20-S009

6. Esposito K, Chiodini P, Bellastella G, Maiorino MI, Giugliano D. Proportion of patients at $\mathrm{HbAlc}$ target $<7 \%$ with eight classes of antidiabetic drugs in type 2 diabetes: systematic review of 218 randomized controlled trials with 78945 patients. Diabetes Obes Metab. 2012;14:228-233.

7. McCreight LJ, Bailey CJ, Pearson ER. Metformin and the gastrointestinal tract. Diabetologia. 2016;59:426-435. doi:10.1007/s00125015-3844-9

8. Dujic T, Zhou K, Tavendale R, Palmer CN, Pearson ER. Effect of Serotonin Transporter 5-HTTLPR polymorphism on gastrointestinal intolerance to metformin: a GoDARTS Study. Diabetes Care. 2016;39:1896-1901. doi:10.2337/dc16-0706

9. Shu Y, Brown C, Castro RA, et al. Effect of genetic variation in the organic cation transporter 1, OCT1, on metformin pharmacokinetics. Clin Pharmacol Ther. 2008;83:273-280. doi:10.1038/sj.clpt.6100275

10. Dujic T, Zhou K, Yee SW, et al. Variants in pharmacokinetic transporters and glycemic response to metformin: a metgen meta-analysis. Clin Pharmacol Ther. 2017;101:763-772. doi:10.1002/cpt.567

11. Zaharenko L, Kalnina I, Geldnere K, et al. Single nucleotide polymorphisms in the intergenic region between metformin transporter OCT2 and OCT3 coding genes are associated with short-term response to metformin monotherapy in type 2 diabetes mellitus patients. Eur J Endocrinol. 2016;175:531-540. doi:10.1530/EJE-160347

12. Xiao D, Guo Y, Li X, et al. The Impacts of SLC22A1 rs594709 and SLC47A1 rs2289669 polymorphisms on metformin therapeutic efficacy in Chinese type 2 diabetes patients. Int $J$ Endocrinol. 2016;2016:4350712. doi:10.1155/2016/4350712

13. Choi JH, Yee SW, Ramirez AH, et al. A common 5'-UTR variant in MATE2-K is associated with poor response to metformin. Clin Pharmacol Ther. 2011;90:674-684. doi:10.1038/clpt.2011.165

14. Tkac I, Klimčáková L, Javorský M, et al. Pharmacogenomic association between a variant in SLC47A1 gene and therapeutic response to metformin in type 2 diabetes. Diabetes Obes Metab. 2013;15:189-191. doi:10.1111/j.1463-1326.2012.01691.x 
15. Chen S, Zhou J, Xi M, et al. Pharmacogenetic variation and metformin response. Curr Drug Metab. 2013;14:1070-1082. doi:10.2174/ 1389200214666131211153933

16. Han TK, Proctor WR, Costales CL, et al. Four cation-selective transporters contribute to apical uptake and accumulation of metformin in Caco-2 cell monolayers. $J$ Pharmacol Exp Ther. 2015;352:519-528. doi:10.1124/jpet.114.220350

17. Liang X, Chien H-C, Yee SW, et al. Metformin is a substrate and inhibitor of the human thiamine transporter, THTR-2 (SLC19A3. Mol Pharm. 2015;12:4301-4310. doi:10.1021/acs.molpharmaceut.5b00501

18. Zhou K. Common variants near ATM are associated with glycemic response to metformin in type 2 diabetes. Nat Genet. 2011;43:117-120.

19. Zhou K, Yee SW, Seiser EL, et al. Variation in the glucose transporter gene SLC2A2 is associated with glycemic response to metformin. Nat Genet. 2016;48:1055-1059. doi:10.1038/ng.3632

20. Rotroff DM, Yee SW, Zhou K, et al. Genetic Variants in CPA6 and PRPF31 are associated with variation in response to metformin in individuals with type 2 diabetes. Diabetes. 2018;67:1428-1440. doi: $10.2337 / \mathrm{db} 17-1164$

21. Florez JC, Jablonski KA, Taylor A, et al. The C allele of ATM rs11212617 does not associate with metformin response in the Diabetes Prevention Program. Diabetes Care. 2012;35:1864-1867. doi:10.2337/dc11-2301

22. van Leeuwen N, Nijpels G, Becker ML, et al. A gene variant near ATM is significantly associated with metformin treatment response in type 2 diabetes: a replication and meta-analysis of five cohorts. Diabetologia. 2012;55:1971-1977. doi:10.1007/s00125-012-2537-X

23. Out M, Becker ML, van Schaik RH, et al. A gene variant near ATM affects the response to metformin and metformin plasma levels: a post hoc analysis of an RCT. Pharmacogenomics. 2018;19:715-726. doi:10.2217/pgs-2018-0010

24. Ji L, Tong X, Wang H, et al. Efficacy and safety of traditional Chinese medicine for diabetes: a double-blind, randomised, controlled trial. PLoS One. 2013;8:e56703. doi:10.1371/journal.pone.0056703

25. Sesti G, Marini MA, Cardellini M, et al. The Arg972 variant in insulin receptor substrate- 1 is associated with an increased risk of secondary failure to sulfonylurea in patients with type 2 diabetes. Diabetes Care. 2004;27:1394-1398. doi:10.2337/diacare.27.6.1394

26. Ren Q, Xiao D, Han X, et al. Genetic and clinical predictive factors of sulfonylurea failure in patients with type 2 diabetes. Diabetes Technol Ther. 2016;18:586-593. doi:10.1089/dia.2015.0427

27. Fagerholm V, Scheinin M, Haaparanta M. alpha2A-adrenoceptor antagonism increases insulin secretion and synergistically augments the insulinotropic effect of glibenclamide in mice. $\mathrm{Br} J$ Pharmacol. 2008;154:1287-1296. doi:10.1038/bjp.2008.186

28. Zhou K, Donnelly L, Burch L, et al. Loss-of-function CYP2C9 variants improve therapeutic response to sulfonylureas in type 2 diabetes: a Go-DARTS study. Clin Pharmacol Ther 2010;87:52-56. doi:10.1038/clpt.2009.176
29. Rena G, Hardie DG, Pearson ER. The mechanisms of action of metformin. Diabetologia. 2017;60:1577-1585. doi:10.1007/s00125017-4342-z

30. Grahame Hardie D. AMP-activated protein kinase: a key regulator of energy balance with many roles in human disease. $J$ Intern Med. 2014;276:543-559. doi:10.1111/joim. 12268

31. Xiao B, Sanders MJ, Carmena D, et al. Structural basis of AMPK regulation by small molecule activators. Nat Commun. 2013;4:3017. doi: $10.1038 /$ ncomms 4017

32. Willows R, Navaratnam N, Lima A, Read J, Carling D. Effect of different gamma-subunit isoforms on the regulation of AMPK. Biochem J. 2017;474:1741-1754. doi:10.1042/BCJ20170046

33. Ross FA, Jensen TE, Hardie DG. Differential regulation by AMP and ADP of AMPK complexes containing different gamma subunit isoforms. Biochem J. 2016;473:189-199. doi:10.1042/BJ20150910

34. Yavari A, Stocker C, Ghaffari S, et al. Chronic Activation of gamma2 AMPK induces obesity and reduces beta cell function. Cell Metab. 2016;23:821-836. doi:10.1016/j.cmet.2016.04.003

35. Jablonski KA, McAteer JB, de Bakker PIW, et al. Common variants in 40 genes assessed for diabetes incidence and response to metformin and lifestyle intervention in the diabetes prevention program. Diabetes. 2010;59:2672-2681. doi:10.2337/db10-0543

36. Ramos-Lopez O, Samblas M, Milagro FI, et al. Circadian gene methylation profiles are associated with obesity, metabolic disturbances and carbohydrate intake. Chronobiol Int. 2018;35:969-981. doi:10.1080/07420528.2018.1446021

37. Maruthur NM, Gribble MO, Bennett WL, et al. The pharmacogenetics of type 2 diabetes: a systematic review. Diabetes Care. 2014;37:876-886. doi:10.2337/dc13-1276

38. Porto AG, Brun F, Severini GM, et al. Clinical Spectrum of PRKAG2 Syndrome. Circ Arrhythm Electrophysiol. 2016;9:e003121. doi:10.1161/CIRCEP.115.003121

39. Luptak I, Shen M, He H, et al. Aberrant activation of AMP-activated protein kinase remodels metabolic network in favor of cardiac glycogen storage. J Clin Invest. 2007;117:1432-1439. doi:10.1172/ JCI30658

40. Hunter RW, Treebak JT, Wojtaszewski JF, Sakamoto K. Molecular mechanism by which AMP-activated protein kinase activation promotes glycogen accumulation in muscle. Diabetes. 2011;60:766-774. doi: $10.2337 / \mathrm{db} 10-1148$

41. Stumvoll M, Nurjhan N, Perriello G, Dailey G, Gerich JE. Metabolic effects of metformin in non-insulin-dependent diabetes mellitus. $N$ Engl J Med. 1995;333:550-554. doi:10.1056/NEJM199508313330903
Pharmacogenomics and Personalized Medicine

\section{Publish your work in this journal}

Pharmacogenomics and Personalized Medicine is an international, peer-reviewed, open access journal characterizing the influence of genotype on pharmacology leading to the development of personalized treatment programs and individualized drug selection for improved safety, efficacy and sustainability. This journal is indexed on the American Chemical Society's Chemical Abstracts Service (CAS). The manuscript management system is completely online and includes a very quick and fair peer-review system, which is all easy to use. Visit http://www.dovepress.com/testimonials.php to read real quotes from published authors. 